\title{
CreaSTEAM. Hacia la mejora de brechas en diversidad mediante la recopilación de proyectos, buenas prácticas y espacios STEAM CreaSTEAM. Towards the improvement of diversity gaps through the compilation of projects, best practices and STEAM spaces
}

\begin{abstract}
David Fonseca ${ }^{1}$, Alicia García-Holgado ${ }^{2}$, Francisco José García-Peñalvo ${ }^{2}$, Elena Jurado ${ }^{1}$, Roger Olivella ${ }^{1}$, Daniel $\mathrm{Amo}^{1}$, Giuseppe Maffeo ${ }^{3}$, Ömer Yigit ${ }^{4}$, Yasin Keskin $^{4}$, Gulay Sevinç ${ }^{5}$, Kirsten Quass $^{6}$, Christian Hofmann ${ }^{7}$

David.fonseca@salle.url.edu aliciagh@usal.es fgarcia@usal.es elena.jurado@salle.url.edu roger.olivella@salle.url.edu daniel.amo@salle.url.edu g.maffeo@fidae.it yigitomer@gmail.com ysnkskn2002@gmail.com gulaysevinc2003@gmail.com kirsten.quass@cbes-lollar.eu christian.hofmann@kultus.hessen.de
\end{abstract}

\author{
${ }^{1}$ GRETEL La Salle \\ Universitat Ramon Llull \\ Barcelona, España
}

${ }^{3}$ FIDAE

Federazione Istituti Di Attività Educative

Roma, Italia

${ }^{5}$ SADETTIN

Sadettin Türkün Ortaokulu

Bursa, Turquia
${ }^{2}$ GRIAL Research Group

Universidad de Salamanca

Salamanca, España

${ }^{4}$ BURSA,

Bursa Il Milli Egitim Mudurlugu

Bursa, Turquia

${ }^{6}$ STUDIENSEMINAR

Studienseminar GHRF

Gießen, Alemania

${ }^{7}$ CLEMENS,

Clemens-Brentano-Europaschule

Gießen, Alemania

\begin{abstract}
Resumen- La falta de diversidad, especialmente la desigualdad de género y el origen social son algunos de los problemas globales que podemos encontrar en diferentes sectores, pero especialmente latentes en el contexto de los campos de la Ciencia, la Tecnología, la Ingeniería (y el recientemente añadido concepto de Artes), y las Matemáticas (STEAM), desde la escuela primaria hasta el nivel universitario y, por tanto, el mercado laboral. Cuando hablamos de diversidad, nos preocupamos por atraer a personas representativas de todos los sectores de la sociedad, diferentes culturas, discapacidades, grupos étnicos, género u orientación sexual. Teniendo en cuenta el contexto descrito, el proyecto CreaSTEAM tiene como objetivo proporcionar un mapa actualizado de los recursos enfocados a la reducción de la brecha de diversidad utilizando enfoques STEAM, y cómo seleccionar de estos recursos las mejores soluciones para crear un marco que permita implementar STEAM-Labs en las escuelas. Los STEAMLabs, como principal novedad del proyecto, fusionan las soluciones que podemos encontrar en los FAB-Labs, MEDIA-Labs, y USERLabs, y nuestro enfoque se centra en la creación de buenas prácticas en estos espacios para promover la diversidad en STEAM, donde la diversidad también abarca la equidad de género, y la inclusión social para la promoción de las vocaciones STEAM.
\end{abstract}

Palabras clave: Brecha en diversidad, STEAM-Lab, buenas prácticas educativas, mapeado de iniciativas educativas, innovación en educación.

Abstract- Lack of diversity, especially gender inequality and social background are some global problems in different sectors but especially latent in the context of the fields of Science, Technology, Engineering (and the recently added concept of Arts), and Mathematics (STEAM), from primary school to university level and, therefore, labor market. When we talk about diversity, we are worried about attracting representative people from all sectors of society, different cultures, disabilities, ethnic groups, gender or sexual orientation. Considering the context described, CreaSTEAM project aims to provide an actualized map of the resources focused on the reduction of the diversity gap using STEAM approaches, and how to select from these resources better solutions to create a framework to implement STEAM-Labs into secondary schools. The STEAM-Labs, as a main innovation of the project, merge the solutions that we can find in FAB-Labs, MEDIA-Labs, and USER-Labs, and our approach is focused on the creation of good practices in these spaces in order to promote diversity in STEAM, where diversity also covers gender equity, and social inclusion for promotion of STEAM vocations.

Keywords: Diversity gap, STEAM-Lab, good educational practices, mapping of educational initiatives, innovation in education.

\section{INTRODUCCIÓN}

Desde los años 80, podemos encontrar numerosos informes, trabajos de innovación y de investigación sobre los problemas de la enseñanza en los campos de ciencias y matemáticas (Bang \& Medin, 2010; Klassen, 2006), así como recomendaciones para mejorar la educación, como el uso del aprendizaje activo, la participación en proyectos y la implantación de la presencia 
de mentores, entre otros muchos aspectos (D. Fonseca \& García-Peñalvo, 2019; David Fonseca, Conde, \& GarcíaPeñalvo, 2018; García Peñalvo \& Colomo Palacios, 2015; Lõpez-Nicolás, Romeo, \& Guerrero, 2014; Petchamé et al., 2020; Romero, Aláez, Amo, \& Fonseca, 2020; SanchezSepulveda, Torres-Kompen, Fonseca, \& Franquesa-Sanchez, 2019; Villegas, Labrador, Fonseca, Fernández-Guinea, \& Moreira, 2019). No obstante, la gran mayoría de estas medidas no se han dirigido a mejorar la situación de los estudiantes con menor presencia en estas áreas como, las mujeres y otras minorías según su origen económico y cultural. La baja diversidad en los estudios STEAM ha sido identificada como uno de los principales problemas a resolver en la actual sociedad, en especial para reducir la brecha de diversidad que existe en estos sectores (Conde et al., 2021; García-Holgado, Camacho Díaz, \& García-Peñalvo, 2019; Garcia-Holgado et al., 2020).

Uno de los principales retos de la Unión Europea es aumentar la participación femenina (identificada como una de las mayores brechas) en STEAM (Astegiano, Sebastián-González, \& Castanho, 2019; Burke \& Mattis, 2007; Chiu, Roy, \& Liaw, 2018; García-Holgado, Verdugo-Castro, González, SánchezGómez, \& García-Peñalvo, 2020; García-Holgado, VerdugoCastro, Sánchez-Gómez, \& García-Pẽalvo, 2019). Además, según las prioridades de la Unión Europea y del SEPIE, la inclusión social es una de las prioridades relevantes de los proyectos ERASMUS + KA2 del 2020 (Jones et al., 2018; Kleijn et al., 2019; Romero et al., 2020). CreaSTEAM aborda dichas problemáticas, no sólo desde la perspectiva de la brecha de género, sino también con un enfoque amplio que abarca la diversidad y la inclusión social como forma de crear espacios inclusivos en los que promover prácticas STEAM (Moreira et al., n.d.; Sáinz, Fàbregues, \& Solé, 2020; Tzu-Ling, 2019; Wang \& Degol, 2017). De este modo, las acciones del proyecto CreaSTEAM apoyarán, a través de las tecnologías digitales y las metodologías pedagógicas innovadoras enfoques con una marcada igualdad de género y de la diversidad a través del concepto STEAM-Lab, un espacio que debe combinar características de otras tipologías específicas de laboratorios profesionales como, por ejemplo, los FAB-LABs, los MEDIALABs, los USER-LABs e incluso cualquier tipo de SOCIALLAB.

En este marco, el proyecto CreaSTEAM (ver Tabla 1), financiado por la Unión Europea a través del programa Erasmus + , introduce un enfoque novedoso para promover la educación el enfoque STEAM en la educación a través de la cooperación interdisciplinaria y la enseñanza, involucrando a diferentes entornos académicos, no sólo a los profesores en activo y a los futuros profesores, sino también a los estudiantes, con diferentes orígenes culturales y socioeconómicos, géneros $\mathrm{y}$, en una segunda etapa, a sus padres, en línea con experiencias previas (David Fonseca, Climent, Canaleta, \& Vicent, 2016; David Fonseca, Climent, Vicent, \& Canaleta, 2016; GarcíaHolgado, Gonzalez-González, Peixoto, Caballero-Gil, \& PlazaMerino, 2020; García-Holgado, González, \& Peixoto, 2019; García-Holgado, Verdugo-Castro, Sánchez-Gómez, \& GarcíaPeñalvo, 2020). En particular, los recursos que compondrán el marco estarán disponibles para ser utilizados no sólo por los profesores en activo sino también por los futuros profesores con el fin de mejorar su formación $\mathrm{y}$ desarrollo profesional relacionados con las competencias en materia de diversidad e inclusión (Weeden, Gelbgiser, \& Morgan, 2020).
Tabla 1. Detalles del proyecto

\begin{tabular}{|c|c|}
\hline Título & $\begin{array}{l}\text { Co-thinking and Creation for STEAM } \\
\text { diversity-gap reduction }\end{array}$ \\
\hline Acrónimo & CreaSTEAM \\
\hline $\begin{array}{l}\text { Entidad } \\
\text { financiadora }\end{array}$ & European Union \\
\hline Llamada & $\begin{array}{l}\text { Erasmus }+ \text { KA2 }- \text { Cooperation and } \\
\text { Innovation for Good Practices. Strategic } \\
\text { Partnerships for higher education }\end{array}$ \\
\hline Referencia & 2020-1-ES01-KA201-082601 \\
\hline $\begin{array}{l}\text { Líder } \\
\text { proyecto }\end{array}$ & David Fonseca \\
\hline $\begin{array}{l}\text { Entidad } \\
\text { coordinadora }\end{array}$ & La Salle, Ramon Llull University \\
\hline Socios & $\begin{array}{l}\text { Universidad de Salamanca (ESP) } \\
\text { Federazione Istituti Di Attività Educative } \\
\text { (ITA) } \\
\text { Bursa Il Milli Egitim Mudurlugu (TUR) } \\
\text { Sadettin Türkün Ortaokulu (TUR) } \\
\text { Clemens-Brentano-Europaschule (ALE) } \\
\text { Studienseminar GHRF Gießen (ALE) }\end{array}$ \\
\hline $\begin{array}{l}\text { Financiación } \\
\text { recibida }\end{array}$ & $240.736,00 €$ \\
\hline $\begin{array}{l}\text { Fecha de } \\
\text { inicio }\end{array}$ & $1 / 10 / 2020$ \\
\hline $\begin{array}{l}\text { Fecha de } \\
\text { finalización }\end{array}$ & $30 / 9 / 2022$ \\
\hline Web & https://creasteam.eu/ \\
\hline
\end{tabular}

En el siguiente apartado se muestra el contexto global del proyecto, para pasar en el apartado 3 a indicar los principales objetivos y fases del proyecto. En la sección 4 mostramos los principales resultados que se esperan obtener para cerrar el artículo con las conclusiones generales del proyecto.

\section{CONTEXTO}

La falta de diversidad es un problema global en diferentes sectores, pero es especialmente latente en el contexto de los campos relacionados con STEM, desde la escuela primaria hasta el nivel universitario y, por tanto, en el mercado laboral. El acceso a los principales estudios y puestos de trabajo relacionados con las Ciencias, Tecnologías, Ingenierías y Matemáticas está claramente dominado por el género masculino. Los estereotipos aprendidos y recogidos a partir de un proceso de permeabilidad sociocultural a través de las interacciones con los modelos más cercanos, como la familia y la escuela son en gran medida causa de dichas problemáticas.

Sin duda, una de las brechas más importantes relacionadas con la diversidad es la brecha de género. Según el informe del National Center for Women Information Technology, solo el $25 \%$ de los puestos de trabajo tecnológicos están ocupados por mujeres. El informe "El futuro de los empleos: Estrategia de empleo, competencias y mano de obra para la cuarta revolución industrial" confirma estos resultados en su capítulo sobre la brecha de género en la industria (Comisión Europea, 2010). Además, el informe destaca que solo el $26 \%$ de los puestos de 
trabajo en el sector tecnológico son desempeñados por mujeres, mientras que las fuerzas del mercado transforman las industrias a favor del desarrollo de las habilidades técnicas. El estudio Trends in International Mathematics and Science Study muestra que sólo el 30\% de las mujeres deciden estudiar carreras STEM (Irvin, Ashcraft, Mclain, \& Eger, 2016). Este problema es más pronunciado en los niveles superiores, tanto en el ámbito académico como en el profesional profesionales. En el contexto europeo, como muestra el Instituto Europeo de la Igualdad de Género, en relación a las personas que se gradúan con una edad entre 25 y 34 años, en estudios terciarios relacionados con el sector STEM en 2016, se observa que la tendencia en los últimos años ha sido hacia una menor representación de las mujeres en comparación con los hombres.

Sin embargo, durante las últimas décadas, las diferentes sociedades y entornos culturales actuales están realizando, en algunos casos, grandes esfuerzos mientras que, en otros pequeños pasos, para lograr una mayor paridad e inclusión de cualquier factor relacionado con el concepto de diversidad (como los factores sociales, económicos o raciales de las personas, su género o cualquier tipo de discapacidad)(Turner, González, \& Wong, 2011).

\section{DESCRIPCIÓN DEL PROYECTO}

\section{A. Objetivos}

El proyecto CreaSTEAM tiene como objetivo principal aumentar el nivel de rendimiento y el interés por las competencias STEAM. Asimismo, el Consorcio (ver Tabla 1) involucra a organizaciones con diferentes perfiles como una forma de desarrollar una asociación entre autoridades escolares regionales, asociaciones y universidades, para colaborar e intercambiar conocimientos para la implementación y la sostenibilidad de los laboratorios STEAM.

Con el trabajo de co-diseño y co-creación de los STEAMLabs en las escuelas y la implementación de píldoras piloto STEAM enfocadas a desarrollar proyectos relacionados con el ambiente cultural de las escuelas, los profesores y los estudiantes, pretendemos involucrar a todas las disciplinas académicas, no sólo a los técnicos, para forzar un cambio en la forma de pensar de los profesores, bajo la hipótesis de que ellos también se han visto afectados por la brecha de género en su formación STEAM previa. Utilizando los STEAM-Labs, podemos fusionar y trabajar en colaboración con todo tipo de materias y contenidos y promover nuevas vocaciones STEAM en alumnos afectados por cualquier tipo de indicador de diversidad (género, social, económica, inmigrantes, discapacidades, etc.). En este sentido, nuestro proyecto tiene los siguientes dos objetivos principales vinculados con los dos resultados intelectuales definidos:

1- Identificar, categorizar y compartir los principales recursos, proyectos y buenas prácticas relacionadas con los enfoques educativos en STEAM (incluyendo el enfoque de las Artes, en línea con las últimas tendencias), con el fin de mejorar cualquier indicador relacionado con la diversidad.

2- Orientar, formar, implementar y evaluar los espacios STEAM (STEAM-Lab) en las escuelas con la complicidad de los centros educativos y el profesorado, para reducir la brecha en las vocaciones STEAM

B. Fases
La duración del proyecto es de 24 meses organizado en cinco fases diferenciadas:

- $\quad$ Fase 1: Mapeado. Con 7 meses de duración (octubre 2020 a abril 2021), coincide con el inicio del objetivo 2 para desarrollar el mapa de iniciativas como primer paso para identificar recursos útiles, como, por ejemplo: comunidades, asociaciones, empresas y organizaciones sin ánimo de lucro que trabajan en la brecha de diversidad en STEAM en los países participantes, etc. Este mapa servirá como entrada y ayuda para el co-diseño de los STEAMLabs (objetivo 1), así como para fomentar las conexiones entre las escuelas y otras iniciativas.

- Fase 2: Co-diseño de los STEAM-Labs (enero - agosto 2021). En esta fase, se cubrirá el co-diseño de los espacios con la participación de todos los socios, no sólo las escuelas, sino también las autoridades (FIDAE, Studienseminar, Bursa MEM) y las universidades (USAL, URL-La Salle). Esta fase tiene tres pasos principales: El primero en que los socios identifican las características de los centros educativos en los que se implantarán los STEAM-Labs (al menos el IES Sadettin Türkün y la Clemens-Brentano-Europaschule, aunque se han establecido 4 centros en Italia y 3 más en España que como escuelas asociadas también seguirán las directrices del proyecto), un segundo paso que se centrará en la formación del profesorado (la cual tendrá un doble objetivo: proporcionar conocimientos y herramientas para la definición de cada STEAM-Lab, y co-diseñar la definición de STEAM-Labs para cubrir las diferentes necesidades de cada institución), y por último, un tercer paso se centrará en la definición final de cada STEAM.

- $\quad$ Fase 3: Despliegue (septiembre 2021 - junio 2022). En esta fase el proyecto se centrará en la puesta en marcha de los STEAM-Labs (Objetivo 1), no sólo la configuración de los espacios y actividades, sino también los pilotos con profesores y alumnos. Esta fase abarca el curso escolar 2021-2022, por lo que cada colegio puede programar su piloto en función de su programa académico.

- Fase 4: Seguimiento. (mayo 2021-agosto 2022). Se desarrollará en paralelo a la $3^{\mathrm{a}}$ fase porque se centrará en la evaluación de los pilotos, así como en la evaluación del perfil del usuario, la motivación, la usabilidad y la satisfacción (en función de las diferentes variables asociadas a la diversidad). Esta fase se inicia cuatro meses antes de la implantación de los STEAM-Labs para preparar el protocolo de evaluación y los instrumentos de recogida de datos y finalizará dos meses después de la finalización de los pilotos para analizar los datos recogidos.

- Fase 5: Integración. (abril - septiembre 2022). En esta fase se realizará la integración de los resultados en el marco de la reducción de la brecha de la diversidad (Objetivo 2). Los datos recogidos durante la fase anterior se utilizarán también para preparar la colección de documentos que componen el marco. En particular, el análisis de los STEAM-Labs permitirá la preparación de paquetes de actividades como selección de las mejores prácticas y actividades que tienen un impacto en el interés de los estudiantes por las áreas STEAM y apoyan la diversidad y la inclusión. Además, durante esta fase, se desarrollará la metodología para implementar los STEAM-Labs utilizando los conocimientos adquiridos en el Objetivo. 


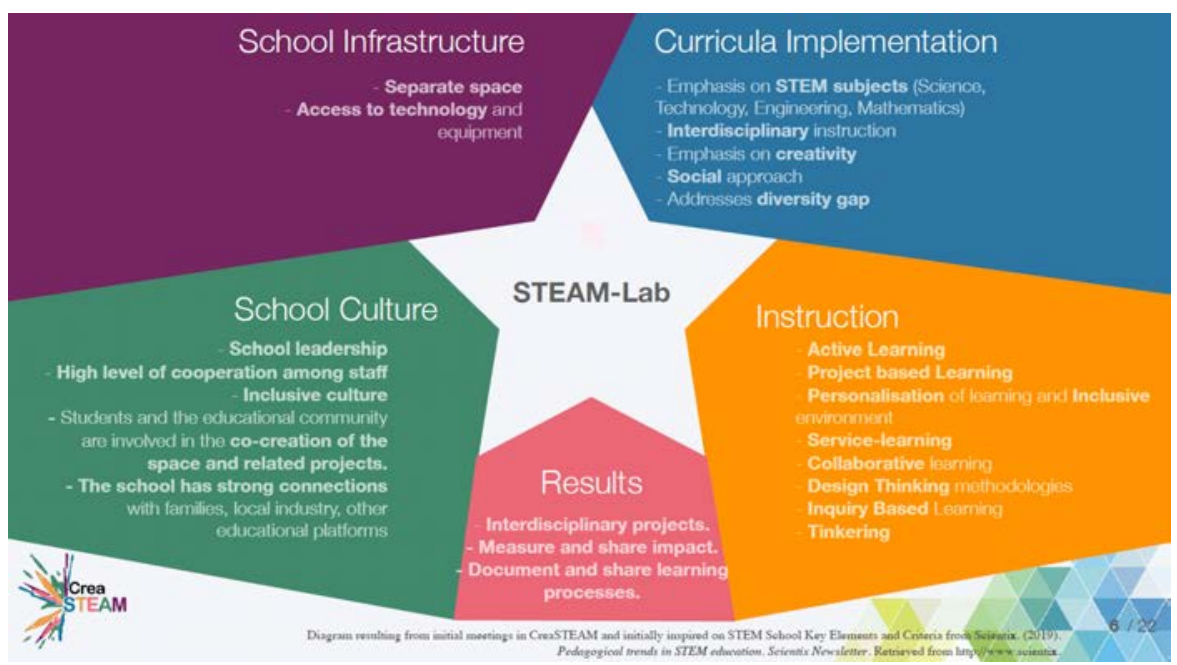

Fig. 1 Principales campos de acción del proyecto.

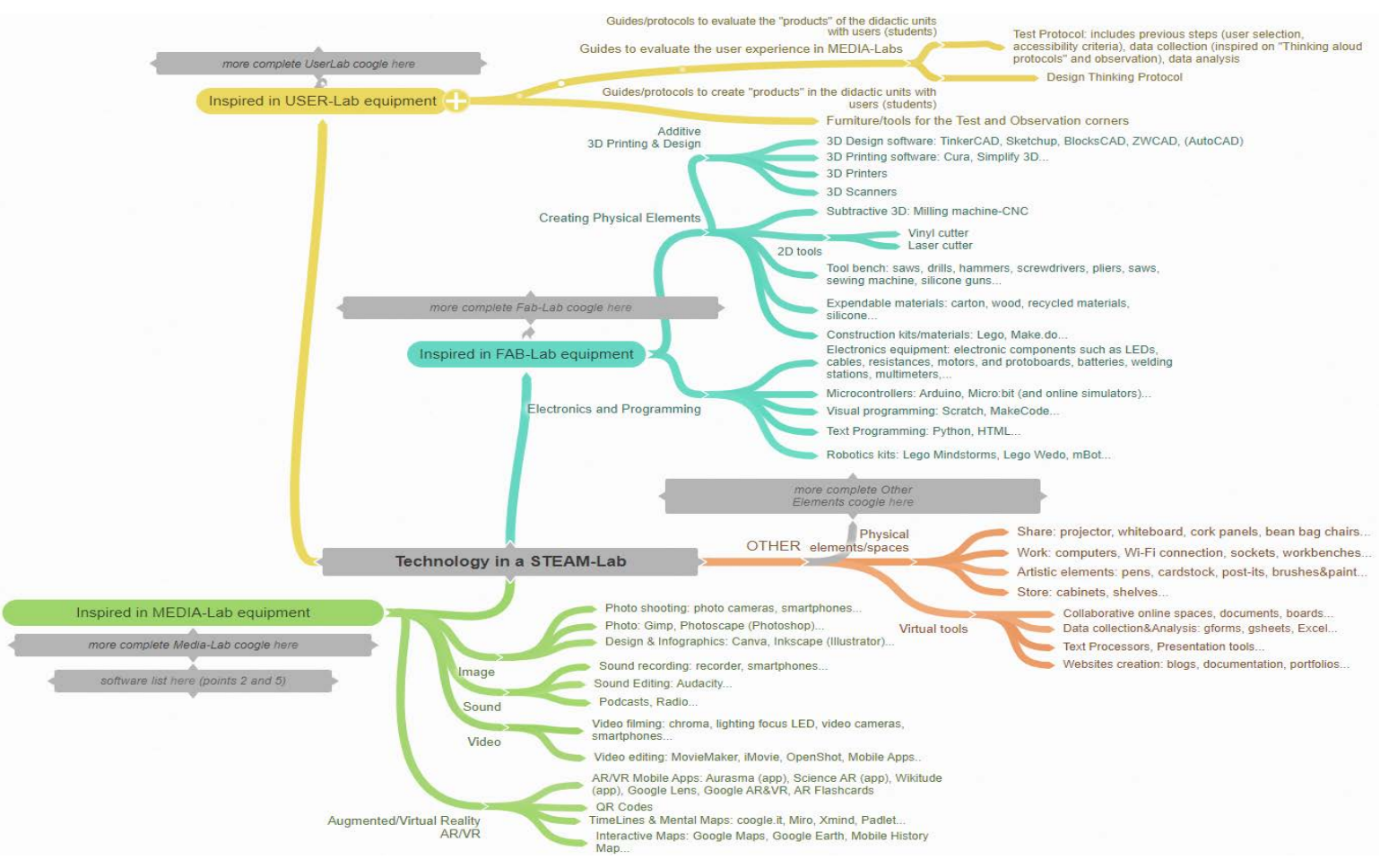

Fig. 2 Diagrama de Tecnologías STEAM-Lab.

\section{Resultados}

Actualmente el proyecto ya ha realizado procesos de conceptualización que se han compartido con los socios y escuelas asociadas para avanzar en la comprensión del concepto STEAM-Lab y cómo aplicar su uso de forma transversal para identificar, solucionar, gestionar o trabajar cualquier tipo de brecha en diversidad, ya sea por su existencia en el contexto de la escuela y/o por ser un objetivo de estudio competencial. En la Fig. 1, podemos observar un primer esquema desarrollado que relaciona los cinco conceptos fundamentales que se están trabajando en el proyecto. Otro de los objetivos realizados se centra la definición de un mapa de herramientas y sistemas que muestren las opciones que un STEAM-Lab puede implementar, fruto de la intersección de espacios como los Fab-lab, los Media-Lab, los User-Lab y los Social-Lab, como se observa en la Fig. 2.
Estos procesos siguen el esquema general del proyecto que conecta en diversos momentos los objetivos y resultados de los dos objetivos principales definidos. Así mismo, otra de las tareas actualmente en proceso de ejecución es el mapa de iniciativas STEAM que atacan problemas de diversidad. En este sentido, a partir de la plantilla de trabajo creada, los socios del proyecto están realizando un mapeado en sus áreas de influencia para identificar dichas iniciativas que pueden ser útiles a la hora de implementar los STEAM-Labs, incluyendo: nombre, web, país, descripción, palabras clave, tipo de proyecto u organización, grupos objetivos, áreas, aspectos relacionados con diversidad, etc. Tal y como hemos indicado, estas iniciativas son parte fundamental del proyecto, ya que cada escuela, en función de sus medios, posibilidades económicas, prioridades educativas, sociales y relaciones con las familias, serán los responsables de implementar espacios y prácticas que generen mejoras en las brechas de diversidad que selecciones, todo ello en comunicación con los padres de los alumnos y 
creando unas guías que permitan su replicación posterior, tanto en el centro como en otras entidades educativas, de forma sostenible.

\section{CONCLUSIONES}

La mayor debilidad identificada era la falta de escuelas donde implantar el STEAM-Lab y los objetivos del proyecto en Italia y España. En este sentido, una vez pasado el primer semestre del proyecto, ya se han conseguido identificar 4 escuelas en Italia, gracias a la labor de FIDAE, y 4 en España mediante la labor de gestión de ARLEP, asociación en la cual se incluyen todas las escuelas de La Salle de España, Portugal y Andorra. Así mismo y fruto de colaboraciones previas, una de las escuelas es ajena a dicha red (Fundació Llor), pero en general la muestra de escuelas permite afirmar que se podrá conseguir una mayor replicación e impacto gracias a la difusión del proyecto y sus resultados por las redes nacionales que gestionan tanto FIDAE en Italia como ARLEP en España y Portugal principalmente. Otros contactos realizados para aumentar el impacto son con entidades como la Asociación de Mujeres Investigadoras y Tecnólogas (AMIT) que forma parte de la Plataforma Europea de Mujeres Científicas (EPWS). También se ha establecido diálogos con Tech\&Ladies, un proyecto español promovido por mujeres organizadoras de los Grupos de Desarrolladores de Google (GDGs) en España. Así mismo se ha reforzado la comunicación y las acciones con el distrito ARLEP como hemos comentado, el cual es la asociación de escuelas lasalianas de España, Portugal y Andorra. Por último, la Autoridad Escolar Estatal de los distritos de Gießen y Vogelsbergkreis (Alemania), una autoridad pública que vela por la calidad del trabajo escolar, la comparabilidad de las calificaciones y la permeabilidad de los programas educativos en sus distritos tiene una relación directa con la escuela alemana que participa en el proyecto y con las instituciones públicas de preparación de profesores (STUDIENSEMINAR). Este socio apoyará la identificación de prácticas y colaborará en la co-creación de los STEAM-Labs para con el fin de asegurar que el enfoque pueda ser replicado en otras escuelas de la región. En Turquia, BURSA está realizando contactos con diversas ONGs y entidades territoriales con el fin de maximizar el impacto del proyecto y las prácticas que se gestionaran en su escuela a cargo (Sadettin). Gracias al soporte de las escuelas y entidades contactadas y al funcionamiento actual del proyecto, se puede afirmar $\mathrm{y}$ conjeturar un progresos sostenible y escalable del trabajo, con un alcance internacional $y$ de largo recorrido que potencialmente deba estudiarse en otros proyectos futuros, tanto en contexto nacional como internacional.

\section{AgradecimiEnTOS}

Con el apoyo del Programa Erasmus+ de la Unión Europea a través de la Acción Clave 2 "Cooperation and Innovation for Good Practices. Strategic Partnerships for school education". Proyecto CreaSTEAM "Co-thinking and Creation for STEAM diversity-gap reduction" (Número de referencia 2020-1-ES01KA201-082601). El contenido de esta publicación no refleja la opinión oficial de la Unión Europea. La responsabilidad de la información y las opiniones expresadas en la publicación recae enteramente en los autores.

\section{REFERENCIAS}

Astegiano, J., Sebastián-González, E., \& Castanho, C. D. T. (2019). Unravelling the gender productivity gap in science: A meta-analytical review. Royal Society Open Science, Vol. 6. https://doi.org/10.1098/rsos.181566

Bang, M., \& Medin, D. (2010). Cultural processes in science education: Supporting the navigation of multiple epistemologies. Science Education, 94(6), 1008-1026. https://doi.org/10.1002/sce.20392

Burke, R. J., \& Mattis, M. C. (2007). Women and minorities in science, technology, engineering and mathematics: Upping the numbers. In Women and Minorities in Science, Technology, Engineering and Mathematics: Upping the Numbers. https://doi.org/10.5860/choice.453752

Chiu, M.-H., Roy, M.-F., \& Liaw, H. (2018). The Gender Gap in Science. Chemistry International, 40(3), 14-17. https://doi.org/10.1515/ci-2018-0306

Comisión Europea. (2010). Un nuevo impulso a la cooperación en educación y formación profesional para apoyar la Estrategia Europa 2020. Retrieved from Bruselas website: http://eur-lex.europa.eu/legalcontent/ES/TXT/PDF/?uri=CELEX:52010DC0296\&fro $\mathrm{m}=\mathrm{EN}$

Conde, M., Rodríguez-Sedano, F. J., Fernández-Llamas, C., Gonçalves, J., Lima, J., \& García-Peñalvo, F. J. (2021). Fostering STEAM through challenge-based learning, robotics, and physical devices: A systematic mapping literature review. Computer Applications in Engineering Education, 29(1), 46-65. https://doi.org/10.1002/cae.22354

Fonseca, D., \& García-Peñalvo, F. J. (2019). Interactive and collaborative technological ecosystems for improving academic motivation and engagement. Universal Access in the Information Society, 18(3). https://doi.org/10.1007/s10209-019-00669-8

Fonseca, David, Climent, A., Canaleta, X., \& Vicent, L. (2016). Evaluación del método Scenario Centered Curriculum en función del perfil tecnológico del estudiante. Education in the Knowledge Society (EKS), 17(3), 67. https://doi.org/10.14201/eks20161736788

Fonseca, David, Climent, A., Vicent, L., \& Canaleta, X. (2016). Learning4work. Designing a new evaluation system based on scenario centered curriculum methodology: The pre-test. Lecture Notes in Computer Science (Including Subseries Lecture Notes in Artificial Intelligence and Lecture Notes in Bioinformatics), 9753, 3-13. https://doi.org/10.1007/978-3-319-39483-1_1

Fonseca, David, Conde, M. Á., \& García-Peñalvo, F. J. (2018). Improving the information society skills: Is knowledge accessible for all? Universal Access in the Information Society, 17(2), 229-245. https://doi.org/10.1007/s10209017-0548-6

García-Holgado, A., Díaz, A. C., \& García-Pẽalvo, F. J. (2019). Engaging women into STEM in Latin America: WSTEM project. ACM International Conference Proceeding Series, 232-239. https://doi.org/10.1145/3362789.3362902

García-holgado, A., Díaz, A. C., \& García-peñalvo, F. J. (2019). La brecha de género en el sector STEM en América Latina: una propuesta europea The gender gap in the STEM sector in Latin America : an European proposal. $V$ Congreso Internacional Sobre Aprendizaje, Innovación y Competitividad, (Cinaic), 704-709. 
https://doi.org/10.26754/cinaic.2019.0143

García-Holgado, A., Gonzalez-González, C. S., Peixoto, A., Caballero-Gil, P., \& Plaza-Merino, P. (2020). Bridging the diversity gap: Actions and experiences fostering diversity in STEM. ACM International Conference Proceeding Series, $126-129$. https://doi.org/10.1145/3434780.3436714

García-Holgado, A., González, C., \& Peixoto, A. (2019). Bridging the diversity gap in STEM. ACM International Conference Proceeding Series, 193-195. https://doi.org/10.1145/3362789.3362948

Garcia-Holgado, A., Mena, J., Garcia-Penalvo, F. J., Pascual, J., Heikkinen, M., Harmoinen, S., ... Amores, L. (2020). Gender equality in STEM programs: A proposal to analyse the situation of a university about the gender gap. IEEE Global Engineering Education Conference, EDUCON, 2020-April, $1824-1830$. https://doi.org/10.1109/EDUCON45650.2020.9125326

García-Holgado, A., Verdugo-Castro, S., González, C., Sánchez-Gómez, M. C., \& García-Peñalvo, F. J. (2020). European Proposals to Work in the Gender Gap in STEM: A Systematic Analysis. Revista Iberoamericana de Tecnologias Del Aprendizaje, 15(3), 215-224. https://doi.org/10.1109/RITA.2020.3008138

García-Holgado, A., Verdugo-Castro, S., Sánchez-Gómez, M. ${ }^{\mathrm{a} C}$, \& García-Peñalvo, F. J. (2020). Facilitating Access to the Role Models of Women in STEM: W-STEM Mobile App. Lecture Notes in Computer Science (Including Subseries Lecture Notes in Artificial Intelligence and Lecture Notes in Bioinformatics), 12205 LNCS, 466-476. https://doi.org/10.1007/978-3-030-50513-4_35

García-Holgado, A., Verdugo-Castro, S., Sánchez-Gómez, M. C., \& García-Pẽalvo, F. J. (2019). Trends in studies developed in Europe focused on the gender gap in STEM. ACM International Conference Proceeding Series. https://doi.org/10.1145/3335595.3335607

García Peñalvo, F., \& Colomo Palacios, R. (2015). Innovative teaching methods in engineering: guest editorial. International Journal of Engineering Education, 31(3), 689-693.

Irvin, A., Ashcraft, C., Mclain, B., \& Eger, E. (2016). WOMEN IN TECH : THE FACTS 2016 UPDATE // See what's changed and what hasn't. National Center for Women \& Information Technology (NCWIT), 102(1), 57-60. Retrieved from https://www.ncwit.org/sites/default/files/resources/wom enintech_facts_fullreport_05132016.pdf

Jones, J., Williams, $\bar{A}$., Whitaker, S., Yingling, S., Inkelas, K., \& Gates, J. (2018). Call to Action: Data, Diversity, and STEM Education. Change: The Magazine of Higher Learning, $\quad 50(2), \quad 40-47$. https://doi.org/10.1080/00091383.2018.1483176

Klassen, S. (2006). Contextual assessment in science education: Background, issues, and policy. Science Education, 90(5), 820-851. https://doi.org/10.1002/sce.20150

Kleijn, D., Bommarco, R., Fijen, T. P. M., Garibaldi, L. A., Potts, S. G., \& van der Putten, W. H. (2019). Ecological Intensification: Bridging the Gap between Science and
Practice. Trends in Ecology and Evolution, Vol. 34, pp. 154-166. https://doi.org/10.1016/j.tree.2018.11.002

Lõpez-Nicolás, G., Romeo, A., \& Guerrero, J. J. (2014). Active learning in robotics based on simulation tools. Computer Applications in Engineering Education, 22(3), 509-515. https://doi.org/10.1002/cae.20576

Moreira, F., Ferreira, M. J., Pereira, C. S., Gomes, A. S., Collazos, C., \& Fonseca, D. (n.d.). ECLECTIC as a learning ecosystem for higher education disruption. Universal Access in the Information Society.

Petchamé, J., Iriondo, I., Riu, D., Masi, T., Almajano, A., \& Fonseca, D. (2020). Project Based Learning or the Rethinking of an Engineering Subject: Measuring Motivation. ACM International Conference Proceeding Series. https://doi.org/10.1145/3434780.3436542

Romero, S., Aláez, M., Amo, D., \& Fonseca, D. (2020). Systematic review of how engineering schools around the world are deploying the 2030 agenda. Sustainability (Switzerland), Vol. https://doi.org/10.3390/su12125035

Sáinz, M., Fàbregues, S., \& Solé, J. (2020). Parent and Teacher Depictions of Gender Gaps in Secondary Student Appraisals of Their Academic Competences. Frontiers in Psychology, https://doi.org/10.3389/fpsyg.2020.573752

Sanchez-Sepulveda, M. V., Torres-Kompen, R., Fonseca, D., \& Franquesa-Sanchez, J. (2019). Methodologies of learning served by virtual reality: A case study in urban interventions. Applied Sciences (Switzerland), 9(23). https://doi.org/10.3390/app9235161

Turner, C. S. V., González, J. C., \& Wong, K. (2011). Faculty women of color: The critical nexus of race and gender. Journal of Diversity in Higher Education, 4(4), 199-211. https://doi.org/10.1037/a0024630

Tzu-Ling, H. (2019). Gender differences in high-school learning experiences, motivation, self-efficacy, and career aspirations among Taiwanese STEM college students. International Journal of Science Education, 41(13), 1870-1884. https://doi.org/10.1080/09500693.2019.1645963

Villegas, E., Labrador, E., Fonseca, D., Fernández-Guinea, S., \& Moreira, F. (2019). Design Thinking and Gamification: User Centered Methodologies. In Lecture Notes in Computer Science (including subseries Lecture Notes in Artificial Intelligence and Lecture Notes in Bioinformatics). https://doi.org/10.1007/978-3-03021814-0_10

Wang, M. Te, \& Degol, J. L. (2017). Gender Gap in Science, Technology, Engineering, and Mathematics (STEM): Current Knowledge, Implications for Practice, Policy, and Future Directions. Educational Psychology Review, Vol. 29, pp. 119-140. https://doi.org/10.1007/s10648015-9355-X

Weeden, K. A., Gelbgiser, D., \& Morgan, S. L. (2020). Pipeline Dreams: Occupational Plans and Gender Differences in STEM Major Persistence and Completion. Sociology of Education, 93(4), 297-314. 